IFT-24/94

\title{
IMMERSIONS AND FOLDS \\ IN STRING THEORIES OF GAUGE FIELDS "̈
}

\author{
Jacek Pawełczyk \\ Institute of Theoretical Physics, Warsaw University, \\ Hoża 69, PL-00-681 Warsaw, Poland.
}

\begin{abstract}
A two-dimensional string model with dynamical cancellation of folds is considered. The action of the model contains the self-intersection number which is defined for surfaces immersed into $4 \mathrm{D}$ targets. The two additional variables are not dynamical and live on a compact manifold. In this sense the model is a compactification of a $4 \mathrm{D}$ theory. The cancellation forces the string $\theta$ angle to be equal $\pi$. Candidates for string states are constructed. Some mathematical background is given.
\end{abstract}

\footnotetext{
* Work supported, in part, by Polish State Committee for Scientific Research (KBN).
} 


\section{Introduction}

It is strongly believed that dynamics of gauge fields can be described in terms of a string theory. The idea was supported by the lattice strong coupling expansion [1] and the $1 / N_{c}$ expansion [2]. The latter applied in 2-dimensional (2D) models gave several well established relations between $\mathrm{QCD}_{2}\left(\right.$ or $\left.\mathrm{YM}_{2}\right)$ and a string theory [3, [1. It appeared that the crucial role is played by the no fold condition, which says that surface-to-surface maps with folds do not contribute to gauge theory functional integrals. This restricts the set of relevant maps, defining a 2D string theory, to a residual set. Moreover, the results indicate that the proper string action should contain the Nambu-Goto term. It is well known that the Nambu-Goto term alone can not give the correct picture, because the appropriate functional integral can not suppress folds.

Recently two solutions to the problem of folds have been proposed [5, 6]. The idea is to construct a topological field theory localized on an appropriate space of maps without folds. In the first work it is the space of holomorphic maps, which corresponds to one chiral sector [4. The another chiral sector is given by anti-holomorphic maps. For completeness of the approach both spaces should be compactified and glued together. This proposal has been almost completely worked out yielding results in accordance with $\mathrm{YM}_{2}$ [4], at least wherever the calculations has been finished. Another proposition deals with the space of harmonic maps [6]. In a sense, it is more natural because holomorphic and anti-holomorphic maps are subsets of harmonic maps. Unfortunately it has not been worked out to this extend as the previous proposal. Despite these successes many properties of string picture of gauge fields are still missing e.g. it is not clear what is the correct 4D model.

In Ref.7 a string theory model with a dynamical cancellation of folds has been proposed. The model consists of two terms: the Nambu-Goto action and a topological term, the self-intersection number [8]. The latter is well defined in a target space of dimension 4, thus two additional variables with values in a $2 \mathrm{D}$ space (farther called vertical) have been introduced. The model properly suppresses folded configurations 
yielding null partition function and null transition amplitudes for microscopic states (infinitesimal punctures) for string propagating on the flat 2D target space-time.

This paper is a continuation of Ref.7. It purpose it two-fold: we want to generalize results in different directions and elaborate on a 1D example, which illustrates most of the basic ideas. We also include some mathematical background. The paper is organized as follows: in Sec.17we describe surface-to-surface maps with special emphasis on some peculiarities of folds and cusps. Section 2 summary facts concerning immersions of surfaces in $4 \mathrm{D}$ spaces. In the next section we dwell upon a $1 \mathrm{D}$ example of nonbacktracking particle. We discuss the functional measure and the propagator on a circle $S^{1}$. Section 4 contains the main results of the paper. In first part we generalize results of Ref.7 for an arbitrary vertical (orientable) manifold. Then a compactification on $S^{2}$ is considered. We show that it forces $\theta=\pi$. Next we discuss shortly $\mathrm{YM}_{2}$ on compact Riemannian surfaces. In final subsection we go to $4 \mathrm{D}$ case and construct string states which bear resemblance with $\mathrm{YM}_{4}$ states. We also give there a general formula for the self-intersection number of a surface with boundary. The last section is devoted to cancellation of singular contributions for 3D string theories.

\section{Surface-to-surface maps.}

Generic surface to surface maps and their singularities were classified by Whitney [9]. Below we briefly summary his results.

Let $X: \Sigma \rightarrow M$ denote a smooth map of a surfaces $\Sigma$ in a 2D manifold $M$ and $S_{1}(X)=\{p \in \Sigma: \operatorname{rank}(d X(p))=1\}$ its set of singular points. In a local coordinate patch the condition means that the matrix of the induced metric $g_{a b}=\partial_{a} X^{\mu} \partial_{b} X^{\mu}$ has one vanishing eigenvalue. For generic maps $S_{1}$ is a $1 \mathrm{D}$ submanifold of $\Sigma$. In particular, if $\Sigma$ is a closed surface (compact, connected, without boundary) then $S_{1}$ is a finite family of disjoint curves (loops) embedded in $\Sigma$.

Farther we define $S_{1,1}=\left\{p \in S_{1}: \operatorname{rank}\left(d\left(\left.X\right|_{S_{1}}(p)\right)=0\right\}\right.$ i.e. $S_{1,1}$ is a set of (disjoint) points at which eigenvector corresponding to zero eigenvalue is tangent to $S_{1}$. 
In a local coordinate system (simple) folds and cusps have the following form: folds, $\left(X^{1}, X^{2}\right)=\left(s^{2}, t\right)$; cusps, $\left(X^{1}, X^{2}\right)=\left(s t-s^{3}, t\right)$.

Let $N$ be a neighborhood of $S_{1}$. $N$ may have topology of a cylinder or a Möbius strip. The second case can happen for non-orientable manifolds. One can think of $\mathrm{N}$ as a (disc) bundle over $S_{1}$. Let $K$ be a line bundle over $S_{1}$ with fibers given by the kernel of $d X$. This bundle can also be orientable (a cylinder) or non-orientable (a Möbius strip). By $w(N), w(K)$ we denote the first Steifel-Whitney classes of the appropriate bundles. Both Stiefel-Whitney classes are in $H^{1}\left(S_{1}, Z_{2}\right)$. Then, of course $w=0$ for the orientable bundle and $w \neq 0$ ( equals to the only non-trivial element of $H^{1}\left(S_{1}, Z_{2}\right)$ ) for the non-orientable bundle. Finally we define the first Steifel-Whitney class of $S_{1,1}$, $w\left(S_{1,1}\right)$, to be the class in $H^{1}\left(S_{1}, Z_{2}\right)$ Poincare dual to the homology class represented by $S_{1,1}$. By this definition $w\left(S_{1,1}\right)$ is zero if the number of points in $S_{1,1}$ is even and it equals to the only non-trivial element of $H^{1}\left(S_{1}, Z_{2}\right)$ otherwise. Here we recall that

addition of two non-trivial elements of $H^{1}\left(S_{1}, Z_{2}\right)$ gives the trivial element due to the mod 2 property.

There is an nice relation between $w(N), w(K)$ and $w\left(S_{1,1}\right)$ [10]: $w(N)+w(K)+$ $w\left(S_{1,1}\right)=0$. It says that for orientable $\Sigma$ (i.e. $w(N)=0$ ) and orientable $K$ there are even number of cusps, while for non-orientable $K$ odd number of cusps. In the next section we shall be mainly interested in the $K$ bundle thus the information whether it is orientable or not will be of some importance.

We know that generic maps do not contribute to the partition function [4]. It means that any string representation of $\mathrm{YM}_{2}$ have to have built in cancellation of these maps.

\section{Immersions}

In the previous section we have described the space of generic surface-to-surface maps $\Sigma \rightarrow M$. Most of these maps are singular thus difficult to work with. The main idea of this work is to desingularize surface-to-surface maps by lifts i.e. maps to an extended 4-dimensional space-time $M \times M_{v}$. The $2 \mathrm{D}$ space $M_{v}$ will be called the vertical space. 
It is known that the space of maps of a $2 \mathrm{D}$ manifold to a $4 \mathrm{D}$ manifold consists mainly of non-singular maps called immersions. The process of lifting is not unique. We shall be able to classify all possible lifts by a topological invariant.

Let us begin with the definition of an immersion. A map $X: \Sigma^{s} \rightarrow \mathcal{M}^{m}$ is an immersion if $\operatorname{rank}(d X)=s$ i.e. the tangent map is of maximal possible rank $(m, s$ denotes dimensions of the spaces, $s \leq m$ ). Roughly speaking it means that the image of $\Sigma^{s}$ in $\mathcal{M}^{m}$ is smooth. It means also that the induced metric $g_{a b} \equiv \partial_{a} \vec{X} \partial_{b} \vec{X}$ is nonsingular. Any map $\Sigma^{s} \rightarrow \mathcal{M}^{m}$ can be approximated by an immersion for $2 s \leq m$ [8, [16]. An immersion can have double points i.e. for some $p \neq q \in \Sigma, X(p)=$ $X(q)$. In the rest of this section we shall consider orientable $\mathcal{M}^{2 s}$ of dimension $2 s$, $s$ even. Hence, generically, double points are isolated and there are no triple points (with an obvious definition). Moreover, the image of $\Sigma^{s}$ intersects transversally i.e. $d X\left(T_{p} \Sigma^{s}\right) \oplus d X\left(T_{q} \Sigma^{s}\right)=T_{X(p)} \mathcal{M}^{2 s}$ where $d X\left(T_{p} \Sigma^{s}\right)$ is the image of the tangent space $\Sigma^{s}$ at $p$ under $d X$. In this case one defines the self-intersection number $I$ to be equal to the number of double points summed with appropriate signs. The latter are chosen in the following manner: (+) if $\left\{d X\left(T_{p} \Sigma^{s}\right), d X\left(T_{q} \Sigma^{s}\right)\right\}$ has the same orientation as $T_{X(p)} \mathcal{M}^{2 s}$ and (-) in the opposite case.

There is a kind of homotopy (regular homotopy) defined in the space of immersions. A regular homotopy is a homotopy which stays to be an immersion for each value of the homotopy parameter. Whitney [8] showed that the intersection number $I$ is invariant under regular homotopies, thus in this sense it is a topological invariant. Moreover $I$ can be an arbitrary integer number 7 and for immersions in the Euclidean spaces $\mathcal{M}^{2 s}=R^{2 s}$, I classify all possible (up to regular homotopies) immersions [15]. In general, immersions $X: \Sigma^{s} \rightarrow \mathcal{M}^{m}$ are classified by monomorphism of tangent bundles $T\left(\Sigma^{s}\right) \rightarrow T\left(\mathcal{M}^{m}\right)$ [15, 25]. The monomorphism is a bundle map which restriction to each fiber of $T\left(\Sigma^{s}\right)$ is a vector space monomorphism i.e. for $\left(v_{1}, \ldots, v_{m}\right) \in T_{p}\left(\mathcal{M}^{m}\right), d X:\left(v_{1}, \ldots, v_{m}\right) \rightarrow$ $\left(d X\left(v_{1}\right), \ldots, d X\left(v_{m}\right)\right)$ is a monomorphism. It is clear that the key role in the classification is played by the space of monomorphisms $T_{p}\left(\Sigma^{s}\right) \rightarrow T_{X(p)}\left(\mathcal{M}^{m}\right)$. If both manifolds have

${ }^{a}$ It is defined mod 2 for non-orientable $\Sigma^{s}$ 
Euclidean signature this is the space of $s$ linearly independent, orthogonal vectors in $R^{m}$ which is so-called Steifel manifold $V_{m, s}=O(m) / O(m-s)$. Inequivalent immersions $S^{s} \rightarrow R^{m}$ are related to elements of $\pi_{s}\left(V_{m, s}\right)$. For $M=S^{2}, N=R^{4} \pi_{2}\left(V_{4,2}\right)=Z$ and elements of $\pi_{2}\left(V_{4,2}\right)$ are self-intersection numbers $I$. The analytic expression for $I$ can be easily found out in the literature [8, 14, 12, 13]. First one defines the normal bundle associated to a given immersion as bundle of orthonormal vectors orthogonal to the image of $\Sigma^{2}$ in $\mathcal{M}^{4}$ under $X$. Its structure group is $S O(2)$. Let the two normals be $\left\{\vec{n}_{1}, \vec{n}_{2}\right\}$. Define a one-form $A=\vec{n}_{1} d \vec{n}_{2}$ and its curvature $F=d A$. Then

$$
I[X]=\frac{1}{2} c_{2}(n)=\frac{1}{4 \pi} \int_{\Sigma} F
$$

i.e. it is half of the Chern number (Euler number) $c_{2}(n)$ of the normal bundle. In the subsection (4.4) we shall generalize this formula for manifolds with boundary.

\section{A non-backtracking particle in 1D space-time.}

In this section we shall consider a simple 1D model of a massive non-backtracking particle. The example will illustrate basic features of the string case of interest.

In the first quantized language the dynamics of a particle moving in 1D target spacetime is given by the ordinary path integral with the action $S[X]=\mu \int_{0}^{1} d t \sqrt{\dot{X}^{2}}$, where $X=X(t)$ is a map from the unit interval $[0,1]$ into the real ax $X \in R$. The set of paths contributing to the path integral contains backtracking paths i.e. paths for which the velocity $\dot{X}$ changes sign. Points where $\dot{X}=0$ will be called singular and denoted by $P_{i}$ ( $i$ enumerate such points). At $P_{i}$ 's the map $X$ ceases to be an immersion. This is a $1 \mathrm{D}$ model of a fold discussed in Sec.1. We assume that points $P_{i}$ are isolated, what is the generic case.

We shall modify the action in such a way that backtracking paths will not contribute to the path integral. The idea is to introduce another field $Y \in R_{v}$ (subscript $v$ means vertical), which will be interpreted as an additional (vertical) coordinate. The map 
$(X(t), Y(t))$ is the lift of $X(t)$. We want to stress that although $X(t)$ maybe singular its lift, generically is an immersion. We supplement the action by a topological term $R$ :

$$
S[X, Y]=\int_{0}^{1} d t \mu \sqrt{\dot{X}^{2}}+i \pi R
$$

where $R=\frac{i}{2 \pi} \int_{0}^{1}(\dot{X} \ddot{Y}-\dot{Y} \ddot{X}) /\left(\dot{X}^{2}+\dot{Y}^{2}\right)$ (for flat space-times) is the rotation number of the vector tangent to the path $(X(t), Y(t))$. In the parameterization $\dot{X}=v \cos \alpha, \dot{Y}=$ $v \sin \alpha, R=\int_{0}^{1} d t \dot{\alpha}(t)$. This formula gives a unique answer if boundary values of $\alpha$ are fixed. Thus we set $Y(0)=Y(1)=\dot{Y}(0)=\dot{Y}(1)=0$ at the ends of paths or $Y=Y_{0}, \dot{Y}(0)=\dot{Y}(1)$ for closed paths. All maps $(X, Y)$ we sum over under the function integral are immersions. This is a dense set of maps in the space of smooth maps.

The model (2) has an enormous group of local symmetries. These are local reparameterizations and $v$-regular homotopies (VRH) $\delta_{y} Y(t)=\epsilon(t)$, where $\epsilon$ is such that $(X, Y+\epsilon)$ is an immersion. Below we describe topological sectors of the model (5) i.e. lifts which differ only by a VRH. Let the path $X(t)$ has folds (see Fig.1a), the latter are characterized by positions of singular points $P_{i}: \dot{X}\left(P_{i}\right)=0$ for all $i$. Because we work with immersions, $\dot{Y}\left(P_{i}\right) \neq 0$ necessarily. Thus either $\dot{Y}\left(P_{i}\right)>0$ or $\dot{Y}\left(P_{i}\right)<0$, because VRH can not change the sign of $\dot{Y}\left(P_{i}\right)$. We can assign the following set of maps to any immersion $f_{i}: P_{i} \rightarrow \operatorname{sign}\left(\dot{Y}\left(P_{i}\right)\right.$ ) (all $i$ ). We shall show that any two lifts are $v$-regularly homotopic (VRH) if and only if they correspond to the same set $\left\{f_{i}\right\}$. If two different lifts $\left(X(t), Y_{1}(t)\right),\left(X(t), Y_{2}(t)\right)$ are VRH then we can make $Y_{1}=Y_{2}$ what defines $\left\{f_{i}\right\}$ uniquely. One the other hand let us assume that two immersions are characterized by the same $\left\{f_{i}\right\}$. Using a VRH one can set $Y_{1}\left(P_{i}\right)=Y_{2}\left(P_{i}\right)$. Thus, both immersions are equal in the infinitesimally small neighborhood of $P_{i}$ 's (up to second power of an infinitesimal quantity) because $\left\{f_{i}\right\}$ gives equality of tangents to both immersions at $P_{i}$ 's. Away from the singular points $X(t)$ is an immersion. In this case the shift parameter $\epsilon(t)$ defined by a VRH can be arbitrary so one can make $Y_{1}(t)=Y_{2}(t)$ there. Thus one can do it everywhere. We conclude that $\left\{f_{i}\right\}$ uniquely defines topological sector of maps. 


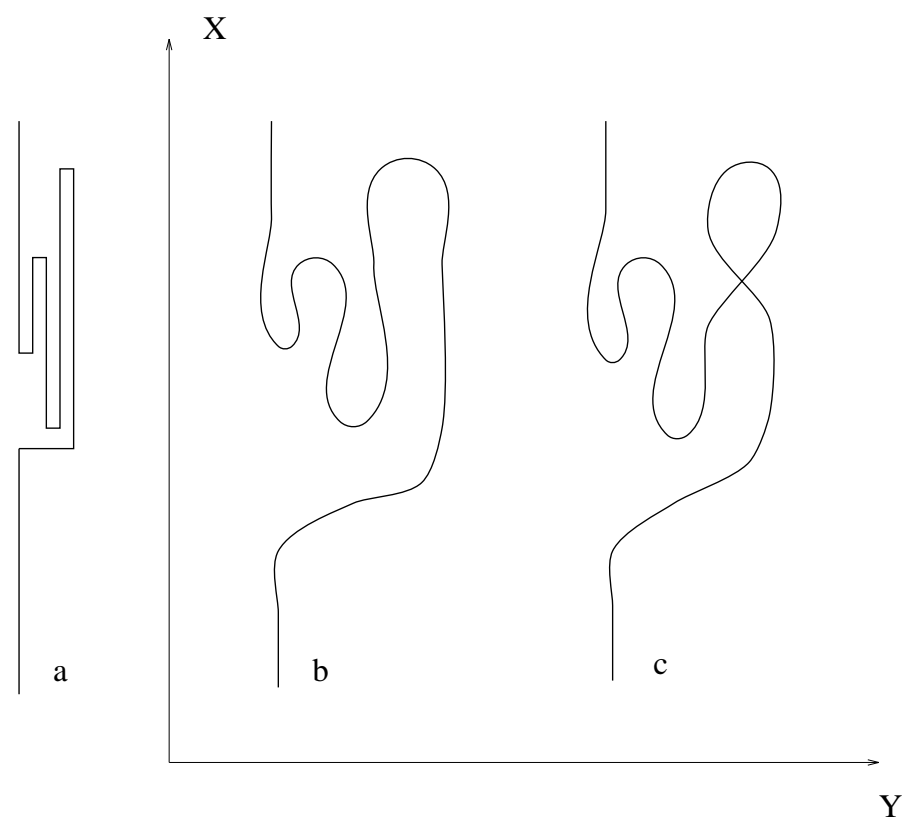

Figure 1: (a) A one dimensional path $X(t)$ with folds. (b,c) Two lifts of the path (a) which rotation numbers differ by one.

We give a heuristic definition of the functional measure. Formally the measure is defined through:

$$
\frac{\mathcal{D} X}{\operatorname{Vol}(\operatorname{Diff})} \frac{\mathcal{D} Y}{\operatorname{Vol}(\mathrm{VRH})}
$$

where $\operatorname{Vol}(\operatorname{Diff})$ is the volume of path diffeomorphisms and $\operatorname{Vol}(\mathrm{VRH})$ is the volume of VRH's. First we note that the space of VRH's depends on $X(t)$, because VRH's are defined by $\delta_{y} Y(t)=\epsilon(t)$, where $\epsilon$ is such that $(X, Y+\epsilon)$ is an immersion. On the other hand the VRH's do not depend on a topological sector of the model i.e. for any choice of $\left\{f_{i}\right\}$ the set of allowed VRH's is the same. Thus we can write the following expression for the measure:

$$
\frac{\mathcal{D} X}{\operatorname{Vol}(\operatorname{Diff})} \frac{\mathcal{D} Y}{\operatorname{Vol}(\mathrm{VRH})}=\frac{\mathcal{D} X}{\operatorname{Vol}(\operatorname{Diff})} F[X] \sum_{\left\{f_{i}= \pm 1\right\}}
$$

where $F[X]$ is a, possible trivial, functional of $X(t)$. The sum runs over different lifts for given singular point $P_{i}$. The cancellation we are going to describe is due to this sum and is independent on $X$. 
After gauging away the VRH the path integral for the model is

$$
\int \mathcal{D} X e^{-S[X]} F[X] \sum_{\left\{f_{i}= \pm 1\right\}} e^{i \pi R\left[\left\{f_{i}\right\}\right]}
$$

It is easy to see that different lifts differ by rotation numbers. An example of a map with folds and two lifts is presented in Fig.1. Lifts which differ at one singular point $P_{i}$ have rotation numbers different by 1 (see Fig.1). Hence their contributions cancel out under the functional integral (5). The sum $\sum_{\left\{f_{i}= \pm 1\right\}}$ is performed independently for each $i$ thus cancelation holds for any set of $P_{i}$ 's. Thus all folds will be suppressed from the functional integration (5). We are left with paths without folds i.e. such that the velocities during the propagation do not change sign. The best way to define the theory without folds is to localize the functional measure on a specific set of paths [5, 6]. This fixes the theory (e.g. $F[X])$ unambiguously.

Below we calculate the propagator of the $1 \mathrm{D}$ non-backtracking particle i.e. the transition amplitude from $X_{0}=X(0)$ to $X_{1}=X(1)$ on the straight line $X \in R$. Boundary conditions for $Y$ are fixed as it has been discussed above. Due to trivial topology of the $X$ space and the reparameterization symmetry only one path contributes. It corresponds to the motion with the constant speed. Thus the transition amplitude is $e^{-\mu l}$, where $\mu$ is the mass of the particle and $l=\left|X_{1}-X_{0}\right|$. If $X \in S^{1}$ then there are also paths winding around the circle. Paths winding $n$ times have the rotation number equal $n$. One can see it mapping the cylinder $S^{1} \times R$ on the plane $R^{2}$ and then calculating the rotation number as in (2). This leads to the following expression for the propagator:

$$
\frac{1}{1+e^{-\mu L}}\left[\theta(l) e^{-\mu l}-\theta(-l) e^{-\mu(l+L)}\right]
$$

where $L$ denotes the circumference of the circle and $l$ is within $\left(-\frac{1}{2} L, \frac{1}{2} L\right)$. The factor $1 /\left(1+e^{-\mu L}\right)$ appears due to the infinite summation over winding sectors with the alternating term $(-1)^{I}$. The relative sign -1 of both contributions comes also from $(-1)^{I}$ : paths contributing to the second term of (6) have rotation numbers different by one (they are accompanied by the additional $e^{-\mu L}$ ). 
The model (5) is in fact a certain compactification of the 2D fermionic particle [17]. If we think of the vertical direction $Y$ as being compactified then its topology should be that of $S^{1}$ instead of $R^{1}$. Proposed above scheme works also in this case but the arguments are a bit more involved. There are infinitely many topological sectors for each singular point due to winding modes around $S^{1}$. Instead of two lifts we get two sets of lifts. Anyway the cancellation holds in a similar manner.

\section{String without folds.}

The string theory functional integral for $2 \mathrm{D}$ targets is a sum over surface-to-surface maps $\Sigma \rightarrow M$, where $\Sigma$ denotes the string world-sheet and $M$ the $2 \mathrm{D}$ target space-time. Surface-to-surface maps were describe in Sec.1. Generic map have singularities: folds and cusps. As works on $\mathrm{QCD}_{2}$ and $\mathrm{YM}_{2}$ shows singular maps do not contribute to physics of these theories [3, 国. Thus we need a mechanism to remove their contribution. Here we will follow the lines of reasoning of Sec.3. In direct analogy with this section we shall introduce a string model and show that for flat target space-times the model suppress generic i.e. folded contributions. What about non-generic surface-to-surface maps? For flat space-times there are no non-singular maps and because the space of maps with folds and cusps is dense in the space of all smooth maps 90 one can safely claim that these maps also do not contribute. Things get more complicated if target space can be arbitrary Riemann surface. Then there are maps without singularities and these can not be simply discarded. We shall discuss these problems in subsections 4.4 .

\subsection{String model}

In this subsection we introduce a string model which suppresses folds. In strict analogy with Sec.3 we introduce two additional, (vertical) world-sheet fields: $\left(Y^{1}, Y^{2}\right) \in M_{v}$ ( $M_{v}$ is a "vertical" surface). The functional integral is over $\left(X^{1}, X^{2}, Y^{1}, Y^{2}\right) \in M \times M_{v}$ configurations. The latter can be viewed as lifts of $\left(X^{1}, X^{2}\right)$ configurations and we know 
from Sec.2 that generically they are immersions. The proposed string action is a direct generalization of the particle case (Sec.3).

$$
S[X]=\mu \int_{M} d^{2} \sigma \sqrt{g}+i \theta I[X, Y]
$$

$I$ is the self-intersection number of the surface immersed in the $4 \mathrm{D}$ space [8, 14, 15, 18, 12, 13. The vertical coordinates enter the action only through $I$. The action (7) is invariant under arbitrary VRH of the vertical fields [8]: $\delta Y^{\mu}(\xi)=\epsilon^{\mu}(\xi),(\mu=1,2)$.

Cancellation of folds for $M=M_{v}=R^{2}$ was presented in Ref.7. Its basic ingredient is the classification of lifts and the alternating sum provided by the self-intersection number $I$. Below we shall generalize these results for arbitrary $M$ and $M_{v}$.

\subsection{Classification of lifts.}

In the following we are going to classify topological sectors of the model. We say that two immersions are in the same topological sector if they can be connected by a VRH. In the following $X$ will denote a map $X: \Sigma \rightarrow M$ with folds and $(X, Y)$ its lift into the extended 4D space-time: $(X, Y): \Sigma \rightarrow M \times M_{v}$, where $M_{v}$ is an arbitrary orientable surface without boundary. In Sec.11 we defined the line bundle $K$ bundle by $d X(K)=0$, where $d X$ is taken at points belonging to $S_{1}$ and the tangent map acts on the fiber over that point. In our case $K$ is trivial bundle $(w(K)=0)$. Lifts of the fold must have non degenerate $2 \mathrm{D}$ tangent space, hence must respect $d Y(K) \neq 0$. In this way the couple $(Y, d Y)$ defines a map (monomorphism) from $K$ to the tangent bundle of $M_{v}\left(T M_{v}\right)$. The set of connected components of such maps, define different topological sectors of lifts. The condition $d Y(K) \neq 0$ imply that this is the same as the set of connected components of maps from $K$ to one dimensional sphere bundle over $M_{v}\left(S M_{v}\right)$. For one fold it is given by $\pi_{1}\left(S M_{v}\right)$, because the fold has topology of $S^{1}$. We conclude that $\pi_{1}\left(S M_{v}\right)$ classify lifts of one fold [20]. Generalization for the case of a map with many folds is obvious, because lifts of folds are independent on each other.

As an example [7] we take first $M=R^{2}=M_{v}$. Then lifts of the i-th fold are classified by $\pi_{1}\left(R^{2} \times S^{1}\right)=Z$. The integer $f_{i} \in \pi_{1}\left(R^{2} \times S^{1}\right)$ is invariant under the 
VRH and is directly related to the self-intersection number $I$ of the lifted configuration. We can see it if we notice that both numbers are additive under gluing [7]. Then the self-intersection number is $I[f]=\sum_{\text {folds }} \pm f_{i}$.

Now we go to the string theory. We want to show that the originally folded configurations $\left(X^{1}, X^{2}\right)$ will cancel out from the partition function. Here one should discussion the construction of the functional integral measure of the theory. The arguments are straightforward generalization of that given in Sec.3 thus we omit them here. We get the following expression for the functional integral:

$$
\int \mathcal{D} X^{1} \mathcal{D} X^{2} e^{-S[X]} F[X] \sum_{\left\{f_{i}\right\}} e^{i \theta I[f]}
$$

The sum over $f_{i}$ 's can be performed independently for each $i$ because $I[f]=\sum_{\text {folds }} \pm f_{i}$. For one fold we get

$$
\sum_{f \in Z} e^{ \pm i \theta f}=2 \pi \delta(\theta)
$$

Thus all folded configurations vanish from the path integral for non-zero $\theta$. Maps contributing to the vacuum-to-vacuum amplitude of the closed string necessarily have folds for the target space $R^{2}$. According to the above discussion the amplitude vanishes. This also holds for any correlation function of any finite set of local operators. Thus the final conclusion of this part of the paper is that the model (7) is trivial for the $R^{2}$ space-time.

The model discussed in this subsection contained one addition parameter compared to $\mathrm{YM}_{2}$ with semisimple Lie group: it is the $\theta$ angle 9 . In the following subsection we shall claim that this $\theta$ is inherited from $\mathrm{YM}_{4}$.

\subsection{Compactification}

One can view (7) as a certain compactification of a $4 \mathrm{D}$ string. Thus $M_{v}$ is a compact manifold without boundary. If a characteristic size of this space is small we expect

\footnotetext{
${ }^{b}$ Strictly speaking the last formula holds if $I=0 \Leftrightarrow f=0$ holds what is true if $w(K)=0$ (see Sec.11). For $w(K) \neq 0, f=0$ may not correspond to $I=0$, because then a surface-to-surface map can not be lifted into $R^{3} 10$. In this case the formula for $I$ may differ by an additive constant (an integer) which is unessential for cancellation of folds (see (9)). This more complicated case was discarded in Ref.7 for the sake of simplicity.

${ }^{c}$ I would like to thank I.Kogan for discussion on this point.
} 
that quantum fluctuations in the compactified directions are strongly suppressed. The standard compactification of $\mathrm{YM}_{4}$ on a 2D torus leads to additional 2D degrees of freedom: the adjoint matter. Its because the $4 \mathrm{D}$ gauge fields $A_{\mu}^{a}\left(x^{1}, \ldots x^{4}\right)(\mu=1, \ldots 4, a$ is the adjoint representation index) decompose into $\left(A_{\alpha}^{a}\left(x^{1}, x^{2}\right), A_{3}^{a}\left(x^{1}, x^{2}\right), A_{4}^{a}\left(x^{1}, x^{2}\right)\right)$ $(a=1,2)$. From the point of view of the uncompactified 2D space-time $A_{3}^{a}, A_{4}^{a}$ are the matter fields. The appearance of the continuous $\theta$ parameter in (9) is natural in this case. It may correspond to the analogous angle in the QCD Lagrangian, because after compactification we have:

$$
\int d^{4} x \operatorname{tr}(F \tilde{F}) \rightarrow 4 \int d^{2} x \epsilon^{\alpha \beta} \operatorname{tr}\left(F_{\alpha \beta} A_{3} A_{4}\right)
$$

In order to get rid of these matter fields (an thus certain ambiguities in the construction) we shall compactify on $S^{2}$.

Topology of the vertical space significantly changes the classification of lifts. Instead of $\mathrm{Z}$ inequivalent regular homotopy sectors we obtain only two. The general arguments presented in the previous subsection say that lifts of one fold are classified by $\pi_{1}\left(S\left(S^{2}\right)\right)=$ $Z_{2}$ [20]. The trivial element of this group corresponds to $I=0$, the non-trivial elelment to $I=1$. Now the cancellation of folds holds only for $\theta=\pi$, as for the particle case. In this way we fixed the value of the only free parameter in the model. It is in accordance with $\mathrm{YM}_{2}$ which for semisimple Lie groups do not have any angle-like parameter.

\section{4 $M$ as a Riemann surface}

In this subsection we shall consider shortly the case when $M$ has non-trivial topology e.g. it is a Riemann surface. First we briefly recapitulate Gross/Washington interpretation of the $1 / \mathrm{N}$ expansion of the $S U(N)$ partition function. The model almost decouple into two (chiral) sectors: orientation preserving and orientation reversing maps. Both sectors are connected only by orientation reversing infinitesimally thin tubes $(\tilde{t})$. One chiral sector looks like branched cover with simple branch points $(i)$. Additionally there are orientation preserving infinitesimally thin tubes $(t)$ connecting different sheets of the 
branched cover and collapsed handles $(h)$. We stress again that $(\tilde{t}),(i),(t),(h)$ maps have singularities of the non-generic type.

Let us note that cancellation of folds holds for arbitrary $\Sigma, M, M_{v}$. It is due to additivity of $I$ under glueing. But this is not the whole story: contrary to $M=R^{2}$ case the space of maps without folds is not empty. Although these maps are not generic we do not have any argument to dismiss them. Maps which do not have singularities obviously have unique lifts. Brenched covers have have $2^{i}$ lifts ( $i$ is the number of branching points). The question is what $I$ have their lifts ? Locally a map with a (simple) branch point at $z=\sigma+i t=0$ looks like $z \rightarrow X=z^{2}$ for $X, z \in C$ and $\operatorname{rank}\left(\left.d X\right|_{z=0}\right)=0$ i.e. $d X$ has two null eigenvectors. It follows that any lift must have two lineary independent tangent vectors at $z=0$ lying in the vertical directions, thus forming an area element of $M_{v}^{2}$. This implies that a branch point can be lifted in two ways differing the orientation of the area form:

$$
\left\{z \rightarrow z^{2}\right\} \stackrel{\text { lift }}{\rightarrow}\left\{z \rightarrow\left(z^{2}, z\right) \text { or }\left(z^{2}, \bar{z}\right)\right\}
$$

The important observation is that branched covers represent elements of the second homology group $H_{2}(M)$ and their lifts elements of $H_{2}\left(M \times M_{v}\right)$. Therefore we can calculate its algebraic self-intersection number defined as below [22]:

$$
a \cdot b=D^{-1}(D(a) \wedge D(b)),
$$

where $D$ denote the Poincare duality (isomorphism), $D: H_{2}\left(M \times M_{v}\right) \rightarrow H^{2}\left(M \times M_{v}\right)$ and $a, b \in H_{2}\left(M \times M_{v}\right)$. Vanishing of $a \cdot a$ implies that there is an embedding of $a$ in $M \times M_{v}$ [21] i.e. $I=0$. If $H_{2}\left(M_{v}\right)=0$ then $H^{4}\left(M \times M_{v}\right)=0$ so $a \cdot a=0$ always. In oreder to simplify consideration for the case $H_{2}\left(M_{v}\right) \neq 0$ we take lifts that do not wind around $M_{v}$. Then $D(a) \in H^{2}(M)$, so $a \cdot a=0$. In all cases we get $I=0$ so the self-intesection term does not influence the functional integral. For example it does not change results of Ref.5 if we treat this work as a realization of (7).

In the end we add few comments about other maps which contribute to the $\mathrm{YM}_{2}$ partition function. Both infinitesimal tubes $t$ and $\tilde{t}$ have singularities along a circle $S^{1}$. 
Tubes $t$ can be viewed as a collision of two branch points [5]. If so they should be lifted with $I=0$. We can not reach this conclusion from the analysis of lifts alone. In fact there are $\mathrm{Z}$ lifts of $t$ for $M_{v}=R^{2}$ because the circel of singularities can be mapped to an arbitrary smooth loop on $M_{v}$. The situation for tubes $\tilde{t}$ is analogous.

We see that the simple approach proposed in this model can not clarify all features of Gross-Taylor interpretation [4]. No wonder it is the case. This goal can be partially achieved by application of more subtle machinery: topological field theory methods. The precise description of one chiral sector of the model can be achieved if the topological theory is localized on holomorphic maps (branched covers) [5]. Tubes $t$ were obtained by a compactification of this space of maps. It is known that the process of compactification is not unique. The other compactifications presumably corresponds to generalized YM theories [26, 27]. Despite these successes the contribution of $\tilde{t}$ tubes have not been completed. Moreover the extension to 4D target space-times is not clear. In this palace we would like to stress that the approach proposed in this paper shows why we should localize on a space of holomorphic or harmonic maps (minimal area maps).

\subsection{D theory and string states}

It is clear that the model (7) has straightforward extensions to 3 and 4 dimensional space-times. It is enough to make the additional dimensions dynamical i.e. add them to the Nambu-Goto action. Higher dimensional string models may require more terms e.g. the extrinsic curvature term [18, 19].

In this subsection we shall dwell upon a problem of description of string states. We define string states to be framed loops in $R^{3}$ [12]. Let $C$ be an oriented, closed curve immersed in $R^{3}$. A framing of $C$ is a field of unit vectors $\vec{n}$ orthogonal to $C$. Then one defines: the (Gauss) linking number $L$, the twist $T$ and the writhe $W$ of the framed curve $C$ as follows [23, 24]:

$$
L\left[C, C^{\prime}\right]=\frac{1}{4 \pi} \int_{C} d X^{i} \int_{C^{\prime}} d Y^{j} \epsilon^{i j k} \frac{\left(X^{k}-Y^{k}\right)}{|X-Y|^{3}}
$$




$$
\begin{aligned}
T\left[C, C^{\prime}\right] & =\frac{1}{2 \pi} \int_{C} d \sigma \epsilon^{i j k} \frac{\dot{X}^{i}}{|\dot{X}|} n^{j} \partial_{\sigma} n^{k} \\
W[C] & =\frac{1}{4 \pi} \int_{C} d X^{i} \int_{C} d Y^{j} \epsilon^{i j k} \frac{\left(X^{k}-Y^{k}\right)}{|X-Y|^{3}}
\end{aligned}
$$

where $C^{\prime}$ is a parallel shift of $C$ by $\epsilon \vec{n}$, where $\epsilon>0$ is an infinitesimal parameter. Using $\vec{Y}=\vec{X}+\epsilon \vec{n}$ for $(\mathbb{1 3})$ one can easily show that : $L\left[C, C^{\prime}\right]=W[C]+T\left[C, C^{\prime}\right]$ (White formula). It follows that for flat curves the writhe is zero thus the linking number equals the twist. The writhe changes by 2 if a curve self-intersects. In order to show it we take two curves: $C$ which nearly self-intersects and $C^{\prime}$ which is a small deformation of $C$ such that $C^{\prime}-C \equiv C_{\varepsilon}=\partial S$ and the surface $S$ intersects $C$ (see Fig.2). We take $S$ to be flat.
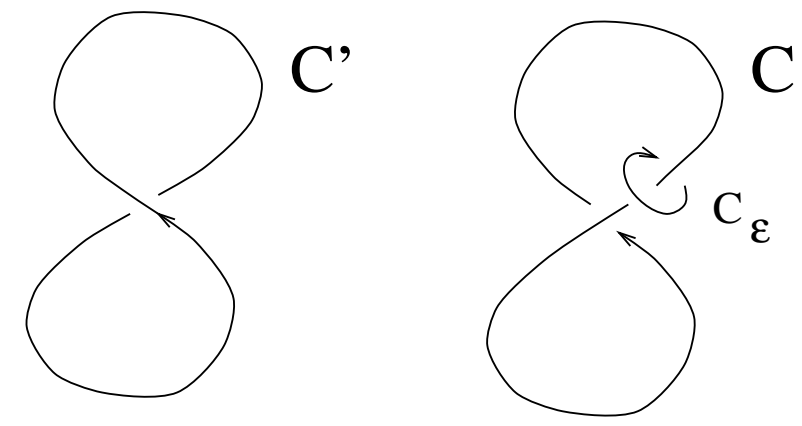

Figure 2: $C^{\prime}-C \equiv C_{\varepsilon}=\partial S$. The small circle around $C$ is $\partial S$.

Then directly from the definition of $W$ we get: $W\left[C^{\prime}\right]-W[C]=2 L\left[C_{\varepsilon}, C\right]+W\left[C_{\varepsilon}\right]$. When $C_{\varepsilon}$ shrinks to zero $L\left[C_{\varepsilon}, C\right]=1$ (with orientation as in Fig.2) and $W\left[C_{\varepsilon}\right]=0$. Thus $W\left[C^{\prime}\right]-W[C]=2$.

Let the target space $\mathcal{M}^{4}$ be a $4 \mathrm{D}$ contractable manifold with boundary $\partial \mathcal{M}^{4}, \Sigma$ a Riemann surface with boundary $\partial \Sigma$ and $X: \Sigma \rightarrow \mathcal{M}^{4}$ an immersion such that $X(\partial \Sigma) \subset$ $\partial \mathcal{M}^{4}$. We take $\mathcal{M}^{4}=R^{3} \times D^{1}\left(D^{1}=\left(t_{i}, t_{f}\right)\right.$ is a time period $)$, so $\partial \mathcal{M}^{4}=R^{3} \cup R^{3}$. Under these conditions we can write down a formula for the self-intersection number of surfaces with boundary. It is given by:

$$
I_{\Sigma, \partial \Sigma}[X]=\frac{1}{2}\left(c_{2}(n)-W[X(\partial \Sigma)]\right)
$$


In (14) the writhe should be calculated with the proper orientation of $\partial \mathcal{M}^{4}$, because $W$ changes sign with change of orientation. $I_{\Sigma, \partial \Sigma}[X]$ is invariant under those regular homotopies of $X$ which do not lead to self-intersections of the boundary. The proof of the formula is very simple. Take a deformations $\delta X$ of $X$. If $\delta X$ has support inside $\Sigma$ then $\delta c_{2}=\frac{1}{2 \pi} \int_{\Sigma} d \delta A=0$ and $\delta W=0$ trivially. Now let $\delta X$ has support which includes $\partial \Sigma$. Because $X(\partial \Sigma) \subset \partial \mathcal{M}^{4}$ both normals are tangent to $\partial \mathcal{M}^{4}$. Let $\sigma$ parameterize $\vec{X}(\partial \Sigma)$ and $\left\{\partial_{\sigma} \vec{X}, \vec{n}_{1}, \vec{n}_{2}\right\}$ be positively oriented then $\vec{n}_{2} \propto\left(\partial_{\sigma} \vec{X} \times \vec{n}_{1}\right)$. Hence we get the following sequence of equalities: $\delta\left(c_{2}(n)-W[X]\right)=\frac{1}{2 \pi} \int_{\Sigma} d \delta A-\delta W=\frac{1}{2 \pi} \int_{\partial \Sigma} \delta\left(\vec{n}_{1} d \vec{n}_{2}\right)-$ $\delta W=-(\delta T+\delta W)=-\delta L=0$. The last equality holds because linking number is invariant under deformations which do not produce self-intersections. If the deformation produces a self-intersection then $W$ changes by 2 (with an appropriate sign convention) changing $I$ by 1 .

If a closed string propagats in the 4D Minkowski space-time the topology of the world-sheet is $S^{1} \times D^{1}\left(D^{1}=\left(t_{i}, t_{f}\right)\right.$ is a time period). Immersions must map the worldsheet time to the target time-like direction. It is easy to see that they are classified by immersions of $S^{1} \rightarrow R^{3}$ which are trivial because $\pi_{1}\left(V_{3,1}\right)=\pi_{1}\left(S^{2}\right)=0$. Hence the normal bundle is trivial and we can take $F=d A$, with globally defined $A$. We define string states as non-self-intersecting framed loops. Any such a state carry an integer quantum number: the linking number $L \in Z$ defined as above. For two such states $\left|L_{1}\right\rangle,\left|L_{2}\right\rangle$ the Minkowski surface connecting them must self-intersects $\left(L_{2}-L_{1}\right)$ times. One can see it noticing that because of triviality of the normal bundle $I_{\Sigma, \partial \Sigma}[X]=$ $1 / 2\left(\int_{\partial \Sigma} A-W\right)=L_{2}-L_{1}$. Thus if a string action contains a $\theta$-term $\left(\theta I_{\Sigma, \partial \Sigma}\right)$ the transition amplitude $\left\langle L_{2} \mid L_{1}\right\rangle$ would be proportional to $\exp \left\{i \theta\left(L_{2}-L_{1}\right)\right\}$. It was climed that the self-intersection number $I$ plays similar role in $4 \mathrm{D}$ string theory as $F \tilde{F}$ in gauge theory 11, 12, 13. If so one sees the full correspondance between framed string states and different topological sectors of QCD. 


\section{Application to 3D YM theory}

We expect that 3D gauge theories also have a string representation. Such systems are interesting by itself but also provide a model for the critical behaviuor of the 3D Ising model about which we comment in the end of this section. In analogy with 2D YangMilles theories one might expect that also 3D theories suppressed singular configurations.

Let us briefly describe the space of maps of a surface $\Sigma$ to 3D Euclidean space $R^{3}$. A generic map is not an immersions but poses singularities: the so-called cross-caps 30] (see Fig.3), where the tangent map drops rank by one. Generically, crosscaps are points $P_{i}$. Any cross-cap ends a line of self-intersections. There is a coordinate system in which

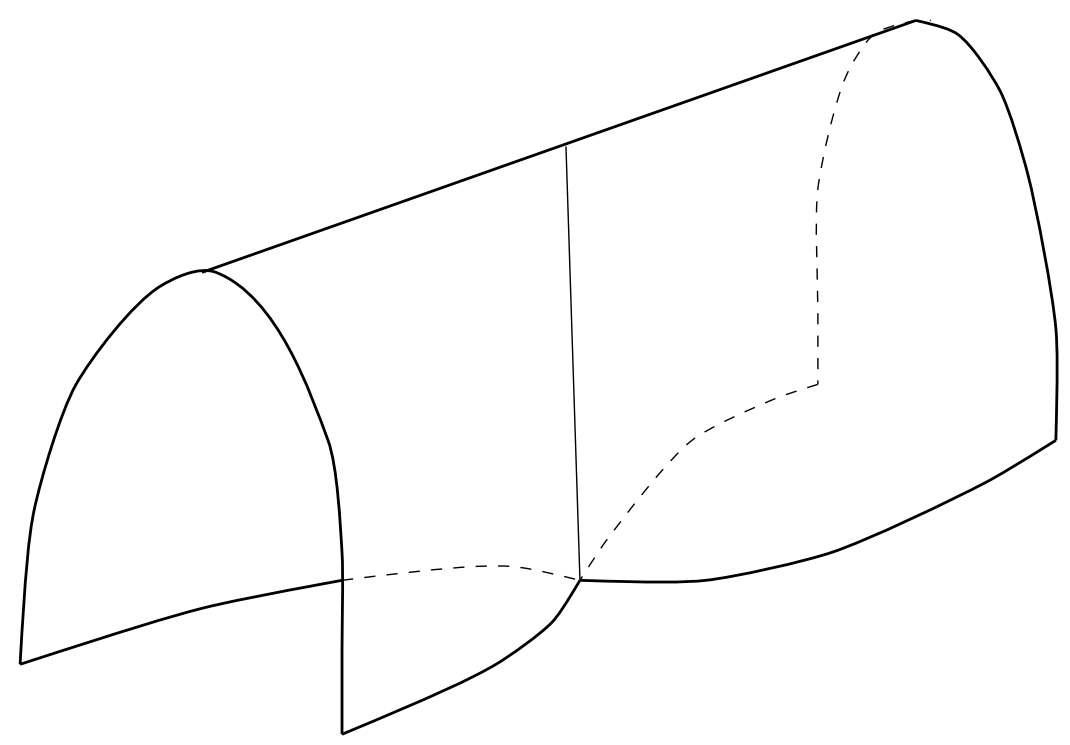

Figure 3: A cross-cap.

the cross-cap has the following form:

$$
(\sigma, t) \rightarrow X=\left(\frac{t^{2}}{4}, \sigma, \frac{\sigma t}{2}\right)
$$

Here the map $X$ is singular at one point $P_{1}=X(\sigma=0, t=0)$, where the vector $\partial_{\sigma} X$ is zero. Fig.3 shows the neighborhood of $P_{1}$. In analogy with 2D Yang-Milles theories one might expect that also 3D theories suppressed singular configurations. One can do it appling a mechanism which is basically the same as that of Sec.⿴囗十. 
We extend the space $R^{3}$ to $R^{4}$ and consider the space of immersions $\Sigma \rightarrow R^{3}$. As a string action we take $S^{(4)}=S^{(3)}+i \pi I$, where $S^{(3)}$ is a $3 \mathrm{D}$ action. $S^{(4)}$ is invariant under VRH's but now the vertical space is one dimensional just as in the 1D particle case, hence the classification of all lifts to $R^{4}$ is the same i.e. they are characterized by $\operatorname{sign}\left(d X^{4}\left(P_{i}\right)\right)=Z_{2}$. It means that to each 3D singular map there corresponds two inequivalent $4 \mathrm{D}$ lifts. Their self-intersection numbers differ by one so their contributions will cancel out from the path integral. We show it for a map $X$ of the sphere $X: \Sigma=S^{2} \rightarrow R^{3}$ having one line of self-intersections ending with two singular points $P_{1}, \quad P_{2}$. If $\operatorname{sign}\left(d X^{4}\left(P_{1}\right)\right)=\operatorname{sign}\left(d X^{4}\left(P_{2}\right)\right)$ the lift has $I=0$. We can see it constructing a homotopy of $X$ which glue $X\left(P_{1}\right)$ with $X\left(P_{2}\right)$. In this case it is a regular homotopy because the tangent map is contineaus and nowhere vanishing. Contrary, for $\operatorname{sign}\left(d X^{4}\left(P_{1}\right)\right) \neq \operatorname{sign}\left(d X^{4}\left(P_{2}\right)\right)$ one can not construct such a regular homotopy so $I \neq 0$. As a convenient realization of the situation we take the following map:

$$
X=\left(\sigma, t-\frac{a t}{\left(1+\sigma^{2}\right)\left(1+t^{2}\right)}, \frac{\sigma t}{\left(1+\sigma^{2}\right)\left(1+t^{2}\right)}\right)
$$

It has two singulat points for $a>1$ which join each other when $a \rightarrow 1$ and finally vanishes for $a<1$. If $X^{4}=t /\left[\left(1+t^{2}\right)\left(1+\sigma^{2}\right)\right]$ then we have the former situation and one can explicitely calculate the self-intersecton number, $I=0$. For $X^{4}=1 /\left[\left(1+t^{2}\right)\left(1+\sigma^{2}\right)\right]$ the self-intersection number is $I=-1$. This argument shows that changing $\operatorname{sign}\left(d X^{4}\left(P_{i}\right)\right)$ changes $I$ by one. Thus including an alternating factor $e^{i \pi I}$ and counting maps as in Sec. 1 we suppress all maps with cross-caps. Thus we are left with immersions into 3D space. It is worth to note that the mechanism works for any $3 \mathrm{D}$ action e.g. $S^{(3)}$ may contain the extrinsic curvature.

Finally we comment on the 3D Ising model. In the dual picture the 3D Ising model is the $3 \mathrm{D} Z_{2}$ gauge theory [28]. It is known [29] that a gas of self-avoiding surfaces in a spatial 3D lattice is in the same universality class as the Ising model. Surfaces we are talking about bound bulks of 3D space in which spins are oriented in the same direction. One expects that the energy of a spin configuration is proportional the area of these surfacee i.e. we expect the action to be proportional to the 3D Nambu-Goto term. The 
problem is how to describe self-avoiding surfaces in a string theory language ? Selfavoiding surfaces are embeddings thus for the 3D Ising model the space of immersions is too large. We need to localize the functional integral on the space of embeddings. Unfortunately it is not known how to do it. A possible solution was proposed few years ago [31, 32] where the cancellation of self-intersecting maps could hold due to summation over topologically different world-sheets. Unfortunately not much has been done in this direction.

Acknowledgment. I would like to thank T. Mostowski for discussions concrerning geometrical aspects of the paper, K.Gawędzki, R.Dijkgraaf, I.Kogan for valuable comments and A.Niemi for kind hospitality in Uppsala University where a part of this paper has been written.

\section{References}

\section{References}

[1] K. Wilson, Phys. Rev. D8, 2445 (1974).

[2] G.'t Hooft, Nucl. Phys. B72, 461 (1974); Nucl. Phys. B75, 461(1974).

[3] W.A.Bardeen, I,Bars, A.J.Hanson and R.D.Peccei, Phys. Rev. D13, 2364 (1976); I.Bars and A.J.Hanson, Phys. Rev. D13, 1744 (1976).

[4] D.J. Gross, Nucl. Phys. B400, 161 (1993); D.J. Gross and W. Taylor, IV, Nucl. Phys. B400, 181 (1993); Nucl. Phys. B403, 395 (1993).

[5] S.Corder, G.Moore and S.Ramgoolam, Yale preprint YCTP-P23-93, hepth/9402107. 
[6] P. Horava, EFI-93-66, hep-th/9311156. To appear in Proc. of The Cargese Workshop, 1993; "Topological Rigid String Theory and Two Dimensional QCD”, PUPT-1547, June 1995, hep-th/9507060.

[7] J. Pawełczyk, Phys. Rev. Lett. 74, 3924 (1995), hep-th/9403175.

[8] H.Whitney, Ann. Math. 45, 220 (1944).

[9] H.Whitney, Ann. Math. 62, 374 (55); M.Golubitsky, V.Guillemin, Stable Mappings and Their Singularities, Springer-Verlag, 1973.

[10] S.J.Blank and C.Curley, Proc. AMS 80, 483 (1980).

[11] A.P.Balachandran, F.Lizzi and G.Sparano, Nucl. Phys. B263, 608 (1986).

[12] P.O.Mazur and V.P.Nair, Nucl. Phys. B 284, 146 (1986).

[13] J.Pawełczyk, Phys. Lett. 311B, 98 (1993).

[14] R.Lashof and S.Smale, Ann. Math. 68, 562 (1958).

[15] S.Smale, Ann. Math. 69, 327 (1959); W.Hirsch, Trans.Amer.Math.Soc.93, 242 (1959).

[16] B.A.Dubrovin, A.T.Fomenko and S.P.Novikov, Modern Geometry - Methods and Applications, vol.II, Springer-Verlag.

[17] A.M.Polyakov, Gauge Fields and Strings, Harwood (1987); A.M.Polyakov, in Fields, Strings and Critical Phenomena, Proc.Les Houches Summer School, Vol. IL, 1988, ed. E.Brezin and J.Zinn-Justin,p North-Holland, Amsterdam, 1990.

[18] A.M.Polyakov, Nucl. Phys. B268, 406 (1986).

[19] L. Peliti and S. Leibler, Phys. Rev. Lett. 54, 1690 (1985); D. Foerster, Phys. Lett. 114A, 115 (1986); H. Kleinert, Phys. Lett. 114A, 263 (1986); H. Kleinert, Phys. Lett. 174B, 335 (1987). 
[20] S.Smale, Trans.Amer.Math.Soc. 87, 492 (1958).

[21] M.Kervaire and J.Milnor, Proc.Nat.Acad.Sci. USA 47, 1651 (1961).

[22] G.E.Bredon, Topology and Geometry, Springer-Verlag, 1993.

[23] J.White, Amer.J.Math.91, 693 (1969); L.H.Kaufmann, Knots and Physics, World Scientific 1993, 2nd edition,Part II sec.15.

[24] J.Grunberg, T.H.Hansson and A.Karlhede, Nucl. Phys. B347, 420 (1990).

[25] M.Adachi, Embeddings and immersions, AMS, 1993.

[26] E.Witten, Comm. Math. Phys 117 (1988) 353; Comm. Math. Phys 118, 411 (1988).

[27] O.Ganor, J. Sonnenschein and S. Yankielowicz, hep-th/9407114, Nucl. Phys. B434 (1995) 139.

[28] R. Savit, Rev. Mod. Phys. 52, 453 (1980).

[29] F.David, Europhys. Lett. 9, 575 (1989).

[30] H.Whitney, Ann. Math. 45, 427 (1944).

[31] J. Distler, Nucl. Phys. B388, 648 (1992).

[32] J.Pawełczyk, Mod. Phys. Lett 9A, 3229 (1994). 